\title{
Optimality for Henig Proper Efficiency in Vector Optimization Involving Dini Set-Valued Directional Derivatives
}

\author{
Guolin Yu, Huaipeng Bai \\ Research Institute of Information and System Computation Science, Beifang University \\ of Nationalities, Yinchuan, China \\ E-mail:guolin_yu@126.com \\ Received March 21, 2011; revised June 8, 2011; accepted June 11, 2011
}

\begin{abstract}
This note studies the optimality conditions of vector optimization problems involving generalized convexity in locally convex spaces. Based upon the concept of Dini set-valued directional derivatives, the necessary and sufficient optimality conditions are established for Henig proper and strong minimal solutions respectively in generalized preinvex vector optimization problems.
\end{abstract}

Keywords: Vector Optimization, Dini Set-Valued Directional Derivative, Generalized Preinvex Function, Henig Proper Efficiency

\section{Introduction}

The study on the optimality conditions for non-smooth and generalized convex vector optimization problem in abstract spaces is a lively subject. Recently, there is a growing interest on this topic by using Dini set-valued directional derivatives. For example: Yang [1] introduced Dini set-valued directional derivatives for a vector valued function in infinite dimensional vector spaces and used this concept to establish the optimality conditions for weakly efficient solution in vector optimization problem under cone-convexity assumption; Ginchev [2] obtained first-order necessary and sufficient optimality conditions in terms of Dini set-valued directional derivatives in finite dimensional linear spaces for locally Lipschitz vector optimization.

It is well known that the concept of convexity and its various generalizations play an important role in operations research and applied mathematics. A meaningful generalized convex function was called the preinvex functions, which was introduced by Weir and Mond [3] and by Weir and Jeyakumer [4] in $n$-dimensional Euclidean space. Nowadays, this class of functions has been extended to the abstract spaces and applied to establish optimality criteria and duality in vector optimization [5-8]. Recently, Qiu [9] in normed linear spaces considered a class of functions called generalized prein- vex and established the unified optimality conditions for set-valued vector optimization problems.

On the other hand, the (weakly) efficient solution is a kind of extremely efficient solutions in vector optimization. Since the range of the set of (weak) efficient solutions is often too large, contracting the solution range is a basic topic in vector optimization. For this purpose, many kinds of proper efficiency have been presented. Among them, an important proper efficiency is called Henig proper efficiency, which was introduced by Henig [10]. It is worthy to notice that the super efficiency, introduced by Borwein [11], equals to the Henig efficiency when the convex cone has a bounded base.

The aim of this paper is to deal with the optimality of Henig proper efficient solutions for vector optimization problems in terms of Dini set-valued directional derivatives under the generalized preinvex assumptions.

\section{Preliminaries}

In this note, it is assumed that $X$ and $Y$ are two locally convex spaces with topological duals $X$ and $Y$, respectively. The partially order of $Y$ is defined by a closed convex cone $C \subset Y$ with $\operatorname{int} C \neq \varphi$. On the other hand, we assume that $\mathrm{Y}$ is a complete vector lattice, i.e., sup $\left\{y_{1}, y_{2}\right\}$ exists for all $y_{1}, y_{2} \in Y$ and every bounded nonempty subset has an infimum and a supre- 
mum. The sets of minimal elements and maximal elements of $K \subset Y$ are defined respectively by

$$
\begin{aligned}
& V-\min K=\{y \in K:(\{y\}-C) \cap C=\{y\}\} \\
& V-\max K=\{y \in K:(\{y\}+C) \cap C=\{y\}\} ;
\end{aligned}
$$

For a set $A \subset Y$, we write

$$
\text { cone }(A)=\{\lambda a: \lambda \geq 0, a \in A\} \text {. }
$$

The closure and interior of a set $A$ are denoted by $c l(A)$ and $\operatorname{int} A$. Let $C^{+}$and $C^{+i}$ be the dual cone and strictly dual cone of convex cone $C^{+}$, defined by

$$
\begin{aligned}
& C^{+}=\left\{f \in Y^{*}: f(y) \geq 0 \text { for all } y \in C\right\}, \\
& C^{+i}=\left\{f \in C^{+}: f(y) \geq 0 \text { for all } y \in C \backslash\{0\}\right\}
\end{aligned}
$$

A nonempty convex subset $B$ of the convex cone $C$ is called a base of $C$, if $C=$ cone $B$ and $0 \notin \mathrm{clB}$. In this paper, it is always assumed that $B$ is a base of $C$. Set

$C^{\Delta}(B)=\left\{f \in C^{+i}:\right.$ There exists $t>0$ such that $f(b) \geq t$, for all $b \in B\}$.

Since $0 \notin c l B$ there exists $\varphi \in Y^{*} \backslash\{0\}$ such that

$$
r=\inf \{\varphi(b): b \in B\}>0 \text {. }
$$

Let

$$
V_{B}=\{y \in Y:|\phi(b)|<r / 2\}
$$
lows:

Define the neighborhood family of $0_{Y}$ in $Y$ as fol-

$\Omega=\left\{U \subset V_{B}: U\right.$ is an open convex circled neighborhood of zero in $\}$.

For each $U \in \Omega$, Let

$$
C_{U}(B)=\text { cone }(B+U)
$$

It has been pointed out in [5] that for each $U \in \Omega$, $C_{U}(B)$ is a pointed convex cone in $Y$ with $C \backslash\{0\} \subset \operatorname{int} C_{U}(B)$.

Definition 2.1. (See [12]) Let $K$ be a nonempty subset of $Y$ and let $B$ be a base of $C . y_{0} \in K$ is said to be a Henig proper efficient point of $K$ with respect to $B$ if there exists $U \in \Omega$ such that

$$
\left(K-y_{0}\right) \cap-\operatorname{int} C_{U}(B)=\varphi
$$

Now, let us recall the concepts of upper and lower Dini set-valued directional derivatives given by Yang [1].

Definition2.2. (See [1]) Let $f: X \rightarrow Y$ be a vector valued function and $x, d \in X$. The limiting set of $f$ at $x$ in the direction $d$ is defined as follows

$$
Y_{f}(x ; d)=\left\{z: z=\lim _{t \rightarrow 0} \frac{f(x+t d)-f(x)}{t}\right\}
$$

For our approach in this note, the following assump- tion will be needed.

Assumption 2.1. (See [1]) The subset $Y_{f}(x ; d)$ has a minimal element and a maximal element.

Definition 2.3. Let $f: X \rightarrow Y$ be a vector valued function. Let $x, d \in X$ be two points. The upper and lower Dini-directional derivatives of $f$ at $x$ in the direction $d$ are defined respectively by

$$
\left\{\begin{array}{l}
f^{+}(x ; d)=V_{-\max } Y_{f}(x ; d) \\
f^{-}(x ; d)=V_{-\min } Y_{f}(x ; d)
\end{array}\right.
$$

Remark 2.1. It is obviously that

$$
\left(f^{+}(x ; d) \cup f^{-}(x ; d)\right) \text {. }
$$

In addition, it has been pointed out in Ref. [1] that if Assumption 2.1 holds, then

$$
f^{+}(x ; d) \neq \phi, f^{-}(x ; d) \neq \phi,
$$

and $f^{+}(x ; d), f^{-}(x ; d)$ as functions of $d$ are positively homogeneous.

Definition 2.4. (See [9]) Let $\eta: X \times X \rightarrow X$ be a map and $\varphi:[0,1] \rightarrow[0,+\infty]$ be a function such that

$$
\lim _{\lambda \rightarrow 0^{+}} \frac{\varphi(\lambda)}{\lambda}=k>0 .
$$

The set $S \subset X$ is called a generalized invex set with respect to $\eta$ and $\varphi$ if for any $x, y \in S$ and any $\lambda \in[0,1]$,

$$
y+\varphi(\lambda) \eta(x, y) \in S .
$$

Suppose that $S \subset X$ is a generalized invex set with respect to $\eta$ and $\varphi$. A vector valued function $f: S \rightarrow Y$ is called generalized $C$-preinvex on $S$ with respect to $\eta$ and $\varphi$ if for any $x, y \in S$ and any $\lambda \in[0,1]$,

$$
\lambda f(x)+(1-\lambda) f(y) \in f(y+\varphi(\lambda) \eta(x, y))+C .
$$

Remark 2.2. It is clear that the concepts of generalized invex sets and generalized preinvex functions are the generalizations of the invex sets and preinvex functions which introduced by Weir [3,4]. In addition, the function $\varphi:[0,1] \rightarrow[0,+\infty]$ in Definition 2.4 has property

$$
\lim _{\lambda \rightarrow 0^{+}} \varphi(\lambda)=0
$$

In fact,

$$
\lim _{\lambda \rightarrow 0^{+}} \varphi(\lambda)=\lim _{\lambda \rightarrow 0^{+}} \frac{\varphi(\lambda)}{\lambda} \cdot \lambda=k \cdot 0=0
$$

We need the next assumptions.

Assumption 2.2. (See [1]) Let $Y_{f}(x ; d)$ be defined as in (2.1). The domination property is said to hold for if

$Y_{f}(x ; d) \subset\left(V_{-\min } Y_{f}(x ; d)+C\right) \cap\left(V_{-\max } Y_{f}(x ; d)-C\right)$ 
The following important property of Generalized cone-preinvex functions will be used in the sequel.

Proposition 2.1. Let $S \subset X$ be a generalized invex set with respect to $\eta$ and $\varphi$ and the vector valued function $f: S \rightarrow Y$ be Generalized $C$ preinvex on $S$ with respect to $\eta$ and $\varphi$. Then for any $x, y \in S$,

$$
f(y)-f(x) \in k Y_{f}(x ; \eta(x, y))+C .
$$

If, in addition, Assumption 2.2 holds, then

$$
f(y)-f(x) \in k f^{-}(x ; \eta(x, y))+C .
$$

Proof: For any $x, y \in S$ and any $\lambda \in[0,1]$, it follows from Definition 2.4 that

$$
\lambda f(y)+(1-\lambda) f(x) \in f(x+\varphi(\lambda) \eta(x, y))+C .
$$

This means that

$$
f(y)-f(x) \in \frac{f(x+\varphi(\lambda) \eta(x, y))-f(x)}{\lambda}+C .
$$

Thus,

$$
f(y)-f(x) \in \frac{\varphi(\lambda)}{\lambda} \cdot \frac{f(x+\varphi(\lambda) \eta(x, y))-f(x)}{\varphi(\lambda)}+C .
$$

Then, it yields from Remark 2.2 that

$$
f(y)-f(x) \in k Y_{f}(x ; \eta(x, y))+C .
$$

Furthermore, if follows from Assumption 2.2 and positive homogeneous property of Dini set-valued directional derivatives that

$$
Y_{f}(x ; \eta(x, y)) \subset V_{-\min } Y_{f}(x ; \eta(x, y))+C .
$$

Thus, we get

$$
f(y)-f(x) \in k f^{-}(x ; \eta(x, y))+C .
$$

\section{Optimality Criteria}

In this section, we apply the Dini set-valued directional derivatives defined in the last section to characterize optimality conditions for a vector optimization problem involving the generalized preinvex functions. We begin by presenting the following vector optimization problem $(V O P)$,

$$
\text { (VOP) } C-\min _{x \in S} f(x)
$$

where $S$ is a nonempty open subset of $X$ and $f: X \rightarrow Y$.

Definition3.1. a) The point is said to be a Henig proper efficient solution of $(V O P)$ with respect to if there exists $U \in \Omega$ such that

$$
(f(S)-f(\bar{x})) \cap\left(-\operatorname{int} C_{U}(B)\right)=\phi .
$$

b) The pointis said to be a strong efficient solution of $(V O P)$, if

$$
f(x)-f(\bar{x}) \in C, \quad \forall x \in S .
$$

Theorem 3.1. Consider the vector optimization problem $(V O P)$.

1) If $\bar{x} \in S$ is a Henig proper efficient of (VOP) with respect to $B$, then there exists $U \in \Omega$ such that

$$
Y_{f}(\bar{x} ; \eta(x, \bar{x})) \cap-\operatorname{int} C_{U}(B)=\phi, \forall x \in S .
$$

In particular,

$$
f^{-}(\bar{x} ; \eta(x, \bar{x})) \cap-\operatorname{int} C_{U}(B)=\phi, \forall x \in S .
$$

2) Assume that $S$ is a generalized invex with respect to $\eta$ and $\varphi$ and $f$ is generalized $C$-preinvex on $S$ with respect to $\eta$ and $\varphi$. If (3.1) holds, then $\bar{x}$ is a Henig proper efficient solution of $(V O P)$.

Proof: 1) If (3.1) does not hold, by the Definition 2.2, then there exists $\hat{x} \in S$ and small enough $\hat{t}>0$ such that $\hat{x}+\hat{t} \eta(\hat{x}+\bar{x}) \in S$ and

$$
f(\hat{x}+\hat{t} \eta(\hat{x}+\bar{x}))-f(\bar{x}) \in-\operatorname{int}\left(C_{U}(B)\right) .
$$

which contradicts to the assumption that $\bar{x} \in S$ is a Henig proper efficient solution of problem $(V O P)$. On the other hand, It is obviously from (2.2) that the inequality (3.2) holds.

2) Suppose that there exists $U \in \Omega$ such that

$$
Y_{f}(\bar{x} ; \eta(x, \bar{x})) \cap-\operatorname{int} C_{U}(B)=\phi, \forall x \in S .
$$

Then

$$
k Y_{f}(\bar{x} ; \eta(x, \bar{x})) \cap-\operatorname{int} C_{U}(B)=\phi, \forall x \in S .
$$

By Proposition 2.1, we get

$$
f(x)-f(\bar{x}) \in k Y_{f}(\bar{x} ; \eta(x, \bar{x}))+C
$$

Noticing that $C \backslash\{0\} \subset \operatorname{int}\left(C_{U}(B)\right)$, we get

$$
f(x)-f(\bar{x}) \in k Y_{f}(\bar{x} ; \eta(x, \bar{x}))+C_{U}(B) .
$$

Hence

$$
f(x)-f(\bar{x}) \cap-\operatorname{int}\left(C_{U}(B)\right)=\phi, \forall x \in S
$$

This means that $\bar{X}$ is a Henig proper efficient solution of the problem (VOP).

Theorem 3.2. Consider the vector optimization Problem $(V O P)$.

1) If $\bar{x} \in S$ is a strong efficient solution of $(V O P)$, then

$$
Y_{f}(\bar{x} ; \eta(x, \bar{x})) \subset C, \forall x \in S .
$$

In particular,

$$
f^{-}(\bar{x} ; \eta(x, \bar{x})) \subset C, \forall x \in S .
$$


2) Assume that $S$ is generalized invex with respect to $\eta$ and $\varphi$ and $f$ is generalized $C$-preinvex on $S$ with respect to $\eta$ and $\varphi$. If (3.3) holds, then $\bar{X}$ is a strong efficient solution of $(V O P)$.

Proof: (1) We proceed by contradiction. Assuming that the condition (3.3) is not satisfied, then there exists $\hat{x} \in S$ such that

$$
Y_{f}(\bar{x} ; \eta(\hat{x}, \bar{x})) \not \subset C .
$$

Thus, there exists small enough $\hat{t}>0$ such that

$$
\hat{x}+\hat{t} \eta(\hat{x}+\bar{x}) \in S \text {. }
$$

and

$$
f(\hat{x}+\hat{t} \eta(\hat{x}+\bar{x}))-f(\bar{x}) \notin C,
$$

which contradicts to that $\bar{x} \in S$ is a strong efficient solution of $(V O P)$. It is easy to obtain inequality (3.4) from the definition of lower Dini directional derivative for $f$.

2) Suppose that the condition (3.3) is satisfied, that is

$$
Y_{f}(\bar{x} ; \eta(x, \bar{x})) \subset C, \forall x \in S .
$$

By Proposition 2.1,

$$
f(x)-f(\bar{x}) \in k Y_{f}(\bar{x} ; \eta(x, \bar{x}))+C \subset C, \forall x \in S
$$

Hence

$$
f(x)-f(\bar{x}) \in C, \forall x \in S .
$$

That is, $\bar{X}$ is a strong efficient solution of problem $(V O P)$.

\section{References}

[1] X. Q. Yang, "A Generalized Upper Dini-Directional Derivative in Vector Optimization," Optimization, Vol. 43, No. 4, 1997, pp. 339-351. doi:10.1080/023319398088443392

[2] I. Ginchev, A. Guerraggio and M. Rocca, "Dini SetValued Derivative in Locally Lipschitz Vector Optimization," Journal of Optimization Theory and Applications, Vol. 142, No. 1, 2009, pp. 87-105. doi:10.1007/s10957-009-9551-2
[3] T. Weir and V. Jeyakumar, "A Class of Nonconvex Functions and Mathematical Programming," Bulletin of Australian Mathematical Society, Vol. 38, No. 2, 1988, pp. 177-189. doi:10.1017/S0004972700027441

[4] T. Weir and B. Mond, "Preinvex Functions in MultipleObjective Optimization," Journal of Mathematical Analysis and Applications, Vol. 136, No. 1, 1988, pp. 29-38. doi:10.1016/0022-247X(88)90113-8

[5] L. Batista Dos Santos, R. Osuna-ómez, M. A.Rojas-Medar and A. Rufián-Lizana, "Preinvex Functions and Weak Efficient Solutions for Some Vectorial Optimization Problem in Banach Spaces," Computers and Mathematics with Applications, Vol. 48, No. 5-6, 2004, pp. 885-895. doi:10.1016/j.camwa.2003.05.013

[6] A. J. V. Brandāo, M. A. Rojas-Medar and G. N. Silva, "Optimality Conditions for Pareto Nonsmooth Nonconvex Programming in Banach Spaces," Journal of Optimization Theory and Applications, Vol. 103, No. 1, 1999, pp. 65-73. doi:10.1023/A:1021769232224

[7] S. K. Mishra, G. Giorgi and S. Y. Wang, "Duality in Vector Optimization in Banach Spaces with Generalized Convexity," Journal of Global Optimization, Vol. 29, pp. 2004, pp. 415-424. doi:10.1016/j.camwa.2007.05.002

[8] G. L. Yu and S. Y. Liu, "Some Vector Optimization Problems in Banach Spaces with Generalized Convexity," Computers and Mathematics with Applications, Vol. 54, No. 11-12, 2007, pp. 1403-1410. doi:10.1016/j.camwa.2007.05.002

[9] J. H. Qiu, "Cone-Directed Contingent Derivatives and Generalized Preinvex Set-Valued Optimization," Acta Mathematica Scientia, Vol. 27, No. 1, 2007, pp. 211-218. doi:10.1016/S0252-9602(07)60019-8

[10] M. I. Henig, "Proper Efficiency with Respect to Cones," Journal of Optimization Theory and Applications, Vol. 36, No. 3, 1982, pp. 387-407. doi:10.1007/BF00934353

[11] J. M. Borwein and D. Zhuang, "Super Efficiency in Vector Optimization," Transactions of the American Mathematical Society, Vol. 338, No. 1, 1993, pp. 105-122. doi: $10.2307 / 2154446$

[12] X. H. Gong, "Optimality Conditions for Henig and Globally Proper Efficient Solutions with Ordering Cone Has Empty Interior," Journal of Mathematical Analysis and Applications, Vol. 307, No. 1, 2005, pp. 12-31. doi:10.1016/j.jmaa.2004.10.001 